\title{
AN EXTENDED CLASS OF TIME-CONTINUOUS BRANCHING PROCESSES
}

\author{
Rong-Rong Chen \\ ( University of Illinois at Urbana-Champaign)
}

\begin{abstract}
This paper is devoted to studying an extended class of time-continuous branching processes, motivated by the study of stochastic control theory and interacting particle systems. The uniqueness, extinction, recurrence and positive recurrence criteria for the processes are presented. The main new point in our proofs is the use of several different comparison methods. The resulting picture shows that the methods are effective and hence should also be meaningful in other situations.
\end{abstract}

\section{INTRODUCTION}

Branching processes (or the Galton-Watson processes) form one of the classical fields of probability theory and have a very wide range of applications. There are several specialized books devoted to the subject (See [2], [3] and [7], for instance). As is well-known, a branching process can be described as follows. Let $\alpha>0$ and let $\left(p_{j}: j \geq 0\right)$ be a probability distribution. Then the process has death rate $\alpha i p_{0}: i \rightarrow i-1(i \geq 1)$ and growth rate $\alpha i p_{j-i+1}: i \rightarrow j(j>$ $i \geq 0)$. Note that the process absorbs at state 0 . Yamazato $(1975)^{[10]}$ considered a modified model, called a return branching process, in which the process starting from 0 can still jump to $j \geq 1$ with rate $q_{0 j}, q_{0}:=\sum_{j \geq 1} q_{0 j}<\infty$. The last model was then analyzed and improved by Pakes and Tavaré (1981) ${ }^{[9]}$. For the reader's convenience and also for later use, we summarize the related previous results ${ }^{[7],[9],[10]}$ as follows.

Theorem 1.1. The branching process is unique iff one of the following conditions holds.

(1) $M_{1}:=\sum_{k=1}^{\infty} k p_{k}<\infty$.

(2) $M_{1}=\infty$ and $\int_{\varepsilon}^{1} \frac{1}{p(s)-s} d s=-\infty$ for some (equivalently, for all) $0<\varepsilon<1$, where $p(s)=\sum_{i=0}^{\infty} p_{i} s^{i}$.

Moreover, when $p_{0}>0$ and $p_{j}>0$ for some $j \geq 2$, the extinction probability of the process is equal to 1 iff $M_{1} \leq 1$.

Next, for the return branching process, the conditions for the uniqueness are the same as above. Finally, assume that the process is unique and irreducible and set $h_{j}=q_{0 j} / q_{0}$.

(4) Then the process is recurrent iff $M_{1} \leq 1$.

(5) It is positive recurrent iff either $M_{1}<1$ and $\sum_{i=1}^{\infty} h_{i} \log i<\infty$ or $M_{1}=1$ but still

$$
\int_{0}^{1} \frac{1-h(s)}{p(s)-s} d s<\infty
$$

where $h(s)=\sum_{i=1}^{\infty} h_{i} s^{i}$.

1991 Mathematics Subject Classification. 60J80, 60J85.

Key words and phrases. Branching processes, uniqueness, recurrence, positive recurrence. 
We remark that the conditions (1) and (2) can be unified into a single condition. That is, the integration given in (2) diverges for $\varepsilon$ sufficiently close to 1 . But the above presentation is more suitable for our purposes.

The present study is motivated by two sources. The first is the stochastic control theory. Recently, Gao (1992) ${ }^{[6]}$ has studied a time-discrete linear controlled branching process. In our context, the model simply replaces the above coefficients $\alpha i$ with $\alpha i+\beta$ for some $\beta \geq 0$ and to take $q_{0 j}=p_{j}$ or $p_{j+1}, j \geq 1$. Here, the appearance of the constant $\beta$ is due to the control. The second source is the interacting particle systems. The dual of a measure-valued process often leads to a modified model of the branching processes. For instance, the following model comes from a typical measure-valued process (the Fleming-Viot process). It was introduced to us by D. A. Dawson: $q_{0 j}=p_{j}(j \geq 1)$ and the coefficient $\alpha i$ is replaced by $i^{2}(i \geq 1)$. The above background leads us to consider an extended class of branching processes with $Q$-matrix:

$$
q_{i j}= \begin{cases}r_{i} p_{j-i+1}, & j \geq i-1 \geq 0 \text { and } j \neq i \\ -r_{i}\left(1-p_{1}\right), & j=i \geq 1 \\ q_{0 j}, & j>i=0 \\ -q_{0}, & j=i=0 \\ 0, & \text { else, } \quad i, j \in \mathbf{Z}_{+} .\end{cases}
$$

where $r_{i} \geq 0$ for all $i \geq 1$. Of course, the typical case we are interested in is where $q_{0 j}=p_{j}$ or $p_{j+1}(j \geq 1)$ and $r_{i}$ is a polynomial with degree $\theta \geq 1$.

Before moving further, let us compare our model with the classical (or the return) branching processes. The $Q$-processes we are considering differ quite a lot from the classical process which absorbs at 0 , and its particles split independently. Hence, the process has a straightforward expression

$$
f_{i j}(t)=\sum_{r_{1}+r_{2}+\cdots+r_{i}=j} f_{1 r_{1}}(t) f_{1 r_{2}}(t) \cdots f_{1 r_{i}}(t)
$$

where $\left(f_{i j}(t)\right)$ is the minimal $Q$-process determined by its $Q$-matrix. As for the return process, by using the method of taboo probability, we still have an explicit expression. However, the formula (1.3) does not hold for our model even in the linear case $\left(r_{i}=\alpha i+\beta, \beta>0\right)$ because when $r_{i} \neq \alpha i$, the particles no longer split independently. Thus, the previous method is useless now. Furthermore, it is impractical to apply the known criteria directly due to the difficulties in solving the corresponding systems of equations or inequalities. Consequently, new methods must be adopted. Throughout this paper, we use several comparison methods, either comparing with the classical branching processes or comparing with some carefully designed birth-death processes. It is interesting that through these methods we still obtain relatively complete results. It is to be hoped that these methods will have more applications in other situations.

We now state the main results of the paper.

Theorem 1.2 (Uniqueness). Let $Q=\left(q_{i j}\right)$ be the $Q$-matrix given by (1.2).

(1) If $M_{1} \leq 1$, then the process is unique.

(2) Let $M_{1} \in(1, \infty)$. Given a non-negative sequence $\left\{w_{k}\right\}$ such that $\sum_{k=1}^{\infty} w_{k} / r_{k}=\infty$. Set $J=\left\{j \geq 1: p_{j+1}>0\right\}$. If

$$
\sup _{i} \sup _{j \in J} \frac{1}{j} \sum_{k=1}^{j} \frac{r_{i} w_{i+k}}{r_{i+k}} /\left(1+\sum_{k=1}^{i} \frac{w_{k}}{r_{k}}\right)<\infty
$$

then the process is unique. Especially, under the assumption $M_{1} \in(1, \infty)$, the conditions are fulfilled (by setting $w_{i}=r_{i}$ and $w_{i} \equiv 1$ respectively) if $r_{i} \leq \alpha i+\beta(i \geq 1)$ for some $\alpha, \beta>0$, or $\sum_{i=1}^{\infty} 1 / r_{i}=\infty$ and $\left\{r_{i}\right\}$ is non-decreasing. 
In particular, if $r_{i} \sim$ const. $i^{\theta}$ and $r_{i}>0$ for all $i \geq 1$, then the process is unique iff

(4) $\theta>1$ and $M_{1} \leq 1$ or

(5) $\theta=1$ and conditions (1) or (2) of Theorem 1.1 hold.

We mention that the uniqueness for the exceptional case of $M_{1}=\infty$ and $\sum_{i=1}^{\infty} 1 / r_{i}=\infty$ is actually indefinite. See for instance part (2) of Theorem 1.1.

The next result can be deduced from Theorem 1.1, it is not new but included here for the sake of thoroughness. We will also present a new and short proof of the theorem, based on the same comparison idea.

Theorem 1.3. Let the process be unique.

(1) (Recurrence). Assume additionally that the process is irreducible. Then it is recurrent iff $M_{1} \leq 1$.

(2) (Extinction). Let $q_{0}=0, p_{0}>0, r_{i}>0$ for all $i \geq 1$ and $p_{k}>0$ for some $k \geq 2$. Then the extinction probability of the process is equal to 1 iff $M_{1} \leq 1$.

Theorem 1.4 (Positive Recurrence). Let the process be irreducible and $M_{1} \leq 1$.

(1) Then the process is positive recurrent if

$$
\sum_{i=1}^{\infty} \frac{1}{r_{i}}<\infty \quad \text { and } \quad \sum_{i=1}^{\infty} h_{i}\left(i \sum_{j=i}^{\infty} \frac{1}{r_{j}}+\sum_{j=1}^{i-1} \frac{j}{r_{j}}\right)<\infty .
$$

The conditions are fulfilled provided $r_{i} \sim$ const. $i^{\theta}$, either $\theta>2$ or $\theta \in(1,2]$ but still $\sum_{i=1}^{\infty} i h_{i}<\infty$.

(2) If $r_{i} \sim$ const. $i^{\theta}(i, \theta \geq 1)$, then the process is positive recurrent iff

$$
\int_{0}^{1} \frac{1-h(s)}{p(s)-s}(1-s)^{\theta-1} d s<\infty
$$

In particular, if $\theta=1$, then the same conclusion of part (5) of Theorem 1.1 holds.

(3) If $\sum_{i} i h_{i}<\infty, M_{1}<1$ and $\underline{\lim }_{i \rightarrow \infty} r_{i} / i>0$, then the process is exponentially ergodic.

As a straightforward consequence of the above theorems, we obtain the following complete result which refers to our typical models.

Corollary 1.5. Let $r_{i} \sim$ const. $i^{\theta}$ for some $\theta \geq 1$ and $r_{i}>0$ for all $i \geq 1$ and take $q_{0 j}=p_{j}$ or $p_{j+1}, j \geq 1$. Then the process is unique iff either $\theta>1$ and $M_{1} \leq 1$, or $\theta=1$ but conditions (1) or (2) of Theorem 1.1 still hold. Next, assume that the process is unique and irreducible. Then it is recurrent iff $M_{1} \leq 1$. Furthermore, it is positive recurrent iff one of the following conditions holds.

(1) $\theta>1$.

(2) $\theta=1$ and $M_{1}<1$.

(3) $\theta=1, M_{1}=1$ and $\int_{0}^{1} \frac{1-s}{p(s)-s} d s<\infty$.

The respective proofs of Theorems 1.2-1.4 are given in the subsequent three sections.

\section{UNIQUENESS}

Proof of Theorem 1.2. a) We show that for the uniqueness, it is enough to consider the case where $q_{0}=0$. To do so, let $\left(\bar{q}_{i j}\right)$ denote for a moment the $Q$-matrix which coincides with our $Q$-matrix $\left(q_{i j}\right)$ except $\bar{q}_{0}=0$. Clearly, the uniqueness of $\left(\bar{q}_{i j}\right)$-process follows from that of the $\left(q_{i j}\right)$-process since the former is dominated by the latter. In detail, the maximal solution of the equation

$$
u_{i}=\sum \frac{q_{i j}}{u} u_{j}, \quad 0 \leq u_{i} \leq 1, \quad i \geq 0
$$


dominates that of the equation

$$
u_{i}=\sum_{j \neq i} \frac{\bar{q}_{i j}}{\lambda+\bar{q}_{i}} u_{j}, \quad 0 \leq u_{i} \leq 1, \quad i \geq 0
$$

Hence the assertion follows from the uniqueness criterion.

Conversely, let the $\left(\bar{q}_{i j}\right)$-process be unique. Then, the corresponding process $\left(\bar{X}_{t}\right)$ has at most a finite number of jumps in every finite time-interval. Now, consider the minimal process $\left(X_{t}\right)$ determined by $\left(q_{i j}\right)$. After $\left(X_{t}\right)$ reaches 0 , it stays at 0 satisfying the exponential law (having parameter $q_{0}$ ) and then jumps to some $j \geq 1$ according to the distribution $\left(h_{j}\right)$. This is the only way in which $\left(X_{t}\right)$ yields more jumps than $\left(\bar{X}_{t}\right)$. Due to the conditional independence of the jumps, the process $\left(X_{t}\right)$ may have at most finitely many more jumps than $\left(\bar{X}_{t}\right)$ in every finite time-interval and so the $\left(q_{i j}\right)$-process should also be unique.

The above observation is due to [9]. Certainly, the same conclusion holds if the single absorbing state 0 is replaced by a finite set of absorbing states.

¿From now on, we ignore $\left(\bar{q}_{i j}\right)$ and simply assume that $q_{0}=0$.

b) To prove part (5) of Theorem 1.2, by a), we may choose $\alpha$ and $\bar{\alpha}$ so that $\alpha i \leq r_{i} \leq \bar{\alpha} i$, $i \geq 1$. Then, the conclusion follows by comparing our process with two classical branching processes with coefficients $\alpha$ and $\bar{\alpha}$ respectively, in the same way as we explained in the first paragraph of a).

c) We now prove parts (1) and (2) of Theorem 1.2. The first part is easy since the process is indeed recurrent as we will see in the next section. An alternative proof goes as follows. Let $\Gamma=\sum_{k=1}^{\infty} k p_{k+1}$. It is easy to check that $\Gamma=M_{1}+p_{0}-1$. Hence $M_{1} \leq 1 \Longleftrightarrow \Gamma \leq p_{0}$ and $M_{1}=1 \Longleftrightarrow \Gamma=p_{0}$. Take $\varphi_{i}=i+1$. Then

$$
\sum_{j} q_{i j}\left(\varphi_{j}-\varphi_{i}\right)=-q_{i, i-1}+\sum_{j=i+1}^{\infty} q_{i j}(j-i)=r_{i}\left(\Gamma-p_{0}\right) \leq 0, \quad i \geq 1 .
$$

Hence $\sum_{j} q_{i j}\left(\varphi_{j}-\varphi_{i}\right) \leq \varphi_{i}$ for all $i \geq 0$. Now, as an application of [4; Theorem 1.11] or [5; Theorem 2.25] with $E_{n}=\{1,2, \ldots, n\}$ and the above choice of $\varphi_{i}$, we obtain the uniqueness of the $Q$-process. Similarly, one can prove part (2) of the theorem with $\varphi_{i}=1+\sum_{k=1}^{i} w_{k} / r_{k}$.

d) It remains to prove part (3). Let $M_{1} \in(1, \infty]$ and $\sum_{i=1}^{\infty} 1 / r_{i}<\infty$. The main idea is to compare our process with a birth-death process. For this, let $\bar{\Gamma} \in\left(p_{0}, \Gamma\right)$, which will be determined later, and consider the birth-death process $\left(\bar{p}_{i j}(t)\right)$ with birth rate $b_{0}=0, b_{i}=r_{i} \bar{\Gamma}$ and death rate $a_{i}=r_{i} p_{0}, i \geq 1$. Since

$$
\sum_{k=1}^{\infty}\left(\frac{1}{b_{k}}+\sum_{i=1}^{k-1} \frac{a_{i+1} \cdots a_{k}}{b_{i} \cdots b_{k}}\right)=\frac{1}{\bar{\Gamma}}\left(\sum_{k=1}^{\infty} \frac{1}{r_{k}}\right) \sum_{k=0}^{\infty}\left(\frac{p_{0}}{\bar{\Gamma}}\right)^{k}<\infty
$$

it follows that $\left(\bar{p}_{i j}(t)\right)$ is not unique. Hence the equation

$$
a_{i}\left(\varphi_{i-1}-\varphi_{i}\right)+b_{i}\left(\varphi_{i+1}-\varphi_{i}\right)=c \varphi_{i} \quad \varphi_{0}=0, c>0, i \geq 1
$$

has a non-negative bounded solution. Moreover, it is easy to check that $\varphi_{i+1} \geq \varphi_{i}$ for all $i \geq 0$. Obviously, we also have

$$
\varphi_{i+1}-\varphi_{i} \geq \frac{p_{0}}{\bar{\Gamma}}\left(\varphi_{i}-\varphi_{i-1}\right), \quad i \geq 1
$$

Next, since

$$
\sum^{\infty} p_{k+1} \sum^{k-1}\left(\underline{\underline{p_{0}}}\right)^{\ell} \uparrow \sum^{\infty} k p_{k+1}=\Gamma \in(0, \infty) . \quad \text { as } \bar{\Gamma} \downarrow p_{0}
$$


we can find some $\bar{\Gamma} \in\left(p_{0}, \Gamma\right)$ so that

$$
\sum_{k=1}^{\infty} p_{k+1} \sum_{\ell=0}^{k-1}\left(\frac{p_{0}}{\bar{\Gamma}}\right)^{\ell} \geq \bar{\Gamma}
$$

By (2.1) and (2.2), we have for $i \geq 1$,

$$
\begin{aligned}
\sum_{j} q_{i j}\left(\varphi_{j}-\varphi_{i}\right) & =a_{i}\left(\varphi_{i-1}-\varphi_{i}\right)+r_{i} \sum_{k=1}^{\infty} p_{k+1} \sum_{\ell=1}^{k}\left(\varphi_{i+\ell}-\varphi_{i+\ell-1}\right) \\
& \geq a_{i}\left(\varphi_{i-1}-\varphi_{i}\right)+r_{i}\left(\varphi_{i+1}-\varphi_{i}\right) \sum_{k=1}^{\infty} p_{k+1} \sum_{\ell=0}^{k-1}\left(\frac{p_{0}}{\bar{\Gamma}}\right)^{\ell} \\
& \geq a_{i}\left(\varphi_{i-1}-\varphi_{i}\right)+b_{i}\left(\varphi_{i+1}-\varphi_{i}\right) \\
& =c \varphi_{i} .
\end{aligned}
$$

By the comparison lemma ([4; Lemma 3.10] and [11; §2 Lemma 1], or [5; Lemma 3.14]) and the uniqueness criterion, we have thus proved that $\left(p_{i j}(t)\right)$ is not unique.

\section{RECURRENCE AND EXtinCtion}

¿From now on, unless otherwise stated, let us assume that the process $\left(p_{i j}(t)\right)$ is unique and irreducible.

Proof of Theorem 1.3. a) We prove that $\left(p_{i j}(t)\right)$ is recurrent provided $M_{1} \leq 1$. Let $\left(\pi_{i j}\right)$ be the embedding chain of $\left(p_{i j}(t)\right)$. It is well known that the $Q$-process $\left(p_{i j}(t)\right)$ is recurrent iff the equation

$$
\sum_{j} \pi_{i j} y_{j} \leq y_{i}, \quad i \geq 1
$$

has a finite solution $\left(y_{i}\right)$ satisfying $\lim _{i \rightarrow \infty} y_{i}=\infty$ (cf. [5; Theorem 4.34 and Theorem 4.24]). Take $y_{i}=i, i \geq 0$. Then, we have

$$
\sum_{j} \pi_{i j} y_{j}=\pi_{i, i-1} y_{i-1}+\sum_{k=1}^{\infty} \pi_{i, i+k} y_{i+k}=i-\frac{1}{1-p_{1}}\left(p_{0}-\Gamma\right) \leq i=y_{i}, \quad i \geq 1 .
$$

We see that $(3.1)$ holds and so $\left(p_{i j}(t)\right)$ is recurrent.

b) We now consider the case that $M_{1}>1$. Clearly, there exists some $0<s_{0}<1$ satisfying $s_{0}>p\left(s_{0}\right)$. To prove the transient assertion of the theorem, the goal is to compare our process with a birth-death process. For this, let $M=p_{0} / s_{0}$ and consider the birth-death process $\left(\bar{p}_{i j}(t)\right)$ with birth rate $b_{i}=(\alpha i+\beta) M$ and death rate $a_{i}=(\alpha i+\beta) p_{0}$. The process $\left(\bar{p}_{i j}(t)\right)$ is certainly unique. It is transient since the equation

$$
\bar{x}_{i}=\sum_{k \neq 0} \bar{\pi}_{i k} \bar{x}_{k}, \quad 0 \leq \bar{x}_{i} \leq 1
$$

has a non-trivial solution: $\bar{x}_{0}=1-s_{0}$ and $\bar{x}_{i}=1-s_{0}^{i}(i \geq 1)$, where $\left(\bar{\pi}_{i j}\right)$ is the embedding chain of $\left(\bar{p}_{i j}(t)\right)([11 ; \S 5$, Lemma 1] or [5; Theorem 4.35]).

On the other hand, by the comparison lemma mentioned above, we know that the equation

$$
x_{i}=\sum_{k \neq 0} \pi_{i k} x_{k}, \quad 0 \leq x_{i} \leq 1
$$

has a non-trivial solution iff the inequality

$$
x_{i} \leq \sum \pi_{i k} x_{k}, \quad 0 \leq x_{i} \leq 1
$$


also has a non-trivial solution. Thus, to prove the transience of $\left(p_{i j}(t)\right)$, it is sufficient to show that $\left(\bar{x}_{i}\right)$ is a solution to (3.2). This can be done by some elementary computations (remember that $s_{0}>p\left(s_{0}\right)$ ).

c) To prove part (2) of the theorem, note that underlying our assumptions, the extinction probability of the $Q$-process $\left(p_{i j}(t)\right)$ is equal to 1 iff the minimal solution of the equation

$$
x_{i}=\sum_{k \neq 0} \pi_{i k} x_{k}+\pi_{i 0}, \quad i \neq 0
$$

is equal to 1 identically. Because the last property is independent of whether the state at 0 is absorptive or not, the proof is exactly the same as those for the recurrence.

\section{Positive Recurrence}

Proof of Theorem 1.4. a) First we prove part (1) of the theorem. Consider the birth-death process $\left(\bar{p}_{i j}(t)\right)$ with rates $b_{i}=a_{i}=r_{i} p_{0}$. The process $\left(\bar{p}_{i j}(t)\right)$ is unique and positive recurrent. Hence, by [8; Theorem 9.4.1] or [1; Theorem 6.2.3], the equation

$$
b_{i}\left(x_{i+1}-x_{i}\right)+a_{i}\left(x_{i-1}-x_{i}\right)+1=0, \quad i \neq 0
$$

has a finite non-negative solution. Without loss of generality, assume that $x_{0}=0$. Then, it is easy to check that a solution to (4.1) is as follows:

$$
x_{i}=\frac{1}{p_{0}}\left(i \sum_{j=i}^{\infty} \frac{1}{r_{j}}+\sum_{j=1}^{i-1} \frac{j}{r_{j}}\right), \quad i \geq 1 .
$$

Clearly, $x_{i}-x_{i-1} \downarrow$ as $i \uparrow$. Thus, for $i \neq 0$,

$$
\begin{aligned}
\sum_{j} q_{i j}\left(x_{j}-x_{i}\right) & \leq q_{i, i-1}\left(x_{i-1}-x_{i}\right)+\sum_{k=1}^{\infty} q_{i, i+k} k\left(x_{i+1}-x_{i}\right) \\
& \leq a_{i}\left(x_{i-1}-x_{i}\right)+b_{i}\left(x_{i+1}-x_{i}\right) \leq 0 .
\end{aligned}
$$

Next, by assumption, we have

$$
\sum_{i=1}^{\infty} q_{0 i} x_{i}=\frac{q_{0}}{p_{0}} \sum_{i=1}^{\infty} h_{i}\left(i \sum_{j=i}^{\infty} \frac{1}{r_{j}}+\sum_{j=1}^{i-1} \frac{j}{r_{j}}\right)<\infty .
$$

Combining these facts with the result just quoted above, we see that $\left(p_{i j}(t)\right)$ is positive recurrent.

b) Next, we prove the main conclusion of part (2).

Because we are in the recurrent situation, up to a positive constant, the equation

$$
\mu_{j} q_{j}=\sum_{i: i \neq j} \mu_{i} q_{i j}, \quad j \geq 0
$$

has a unique positive solution.

i) We show that when $r_{i}=i^{\theta}(i, \theta \geq 1),\left(\mu_{i}\right)$ is bounded. First, we have

$$
\mu_{2}=\frac{\mu_{1} q_{1}-\mu_{0} q_{01}}{q_{21}} \leq \frac{\mu_{1} q_{1}}{q_{21}}=\mu_{1} \frac{1-p_{1}}{2^{\theta} p_{0}} \leq \mu_{1} .
$$

Assume that $\mu_{j} \leq \mu_{j-1} \leq \cdots \leq \mu_{1}$. Then for $j \geq 2$, we have 
Notice that

$$
\begin{aligned}
q_{j+1, j}-q_{j}+\sum_{i=1}^{j-1} q_{i j} & =p_{0}\left[(j+1)^{\theta}-j^{\theta}\right]-\sum_{k=2}^{j}\left[j^{\theta}-(j+1-k)^{\theta}\right] p_{k}-j^{\theta} \sum_{k=j+1}^{\infty} p_{k} \\
& \geq \theta j^{\theta-1} p_{0}-\theta j^{\theta-1} \sum_{k=2}^{j}(k-1) p_{k}-\theta j^{\theta-1} \sum_{k=j+1}^{\infty}(k-1) p_{k} \\
& =\theta j^{\theta-1}\left(p_{0}-\Gamma\right) \geq 0,
\end{aligned}
$$

here the inequality comes from the mean value theorem. We obtain $\mu_{j+1} \leq \mu_{j}$. By induction, we have proved that $\left(\mu_{i}\right)$ is bounded.

ii) Assume that $\sum_{j=1}^{\infty} \mu_{j} r_{j} s^{j}<\infty$ for every $0 \leq s<1$. Since

$$
\mu_{j} q_{j}=\sum_{i=0}^{j-1} \mu_{i} q_{i j}+\mu_{j+1} q_{j+1, j}, \quad j \geq 1
$$

we have

$$
\mu_{j} r_{j}\left(1-p_{1}\right)=\mu_{0} q_{0 j}+\sum_{i=1}^{j-1} \mu_{i} r_{i} p_{j-i+1}+\mu_{j+1} r_{j+1} p_{0}, \quad j \geq 1 .
$$

If we multiply both sides of (4.2) by $s^{j}$, then sum both sides over $j \geq 1$, we find that

$$
\sum_{j=1}^{\infty} \mu_{j} r_{j} s^{j-1}=\mu_{0} q_{0} \frac{1-h(s)}{p(s)-s}
$$

iii) We now prove the main assertion of part (2) in the particular case that $r_{j}=j^{\theta}(j, \theta \geq 1)$. From $i$ ) and $i i$ ), it follows that

$$
\sum_{j=1}^{\infty} \mu_{j} j^{\theta} s^{j-1}=\mu_{0} q_{0} \frac{1-h(s)}{p(s)-s} .
$$

If we multiply both sides of (4.4) by $(1-s)^{\theta-1}$ and integrate from 0 to 1 , we obtain

$$
\sum_{j=1}^{\infty} \mu_{j} j^{\theta} \int_{0}^{1} s^{j-1}(1-s)^{\theta-1} d s=\mu_{0} q_{0} \int_{0}^{1} \frac{1-h(s)}{p(s)-s}(1-s)^{\theta-1} d s .
$$

Noticing that

$$
\int_{0}^{1} s^{j-1}(1-s)^{\theta-1} d s=B(j, \theta)=\frac{\Gamma(j) \Gamma(\theta)}{\Gamma(j+\theta)}
$$

and using Stirling formula, we get

$$
j^{\theta} B(j, \theta) \sim j^{\theta} \Gamma(\theta) \frac{j^{j-1 / 2} e^{-j}}{(j+\theta)^{j+\theta-1 / 2} e^{-j-\theta}} \sim \text { const. }, \quad j \rightarrow \infty .
$$

We have thus proved that $\sum_{j=1}^{\infty} \mu_{j}<\infty$ iff

$$
\sum_{j=1}^{\infty} \mu_{j} j^{\theta} B(j, \theta)=\mu_{0} q_{0} \int_{0}^{1} \frac{1-h(s)}{p(s)-s}(1-s)^{\theta-1} d s<\infty .
$$


$i v)$ For general $r_{i} \sim$ const. $i^{\theta}(i, \theta \geq 1)$, choose $c_{0}, c_{1}>0$ such that $c_{0} i^{\theta} \leq r_{i} \leq c_{1} i^{\theta}$. Recall that an irreducible recurrent process $\left(p_{i j}(t)\right)$ with $Q$-matrix $\left(q_{i j}\right)$ is positive recurrent iff the minimal solution $\left(x_{i}^{*}\right)$ of the equation

$$
x_{i}=\sum_{j \neq i, 0} \frac{q_{i j}}{q_{i}} x_{j}+\frac{1}{q_{i}}, \quad i \geq 0
$$

is finite ([8; Theorem 9.4.1]). Next, let $\left(x_{i}^{c *}\right)$ denote the minimal solution of (4.5) replacing $r_{i}$ with $c i^{\theta}$. Then, by the comparison theorem ([8; Theorem 3.3.1] or [4; Theorem 2.5]), we have

$$
x_{i}^{c_{0} *} \geq x_{i}^{*} \geq x_{i}^{c_{1} *}, \quad i \geq 0 .
$$

Next, by the linear combination theorem ([8; Theorem 3.3.2] or [4; Theorem 2.4]),

$$
c_{0} x_{i}^{c_{0} *}=c_{1} x_{i}^{c_{1} *}=x_{i}^{1 *}, \quad i \geq 0 .
$$

Hence, $x_{i}^{c_{0} *}, x_{i}^{*}, x_{i}^{c_{1} *}$ and $x_{i}^{1 *}$ are all finite or infinite simultaneously. We have thus reduced the general case to the particular one treated above.

c) Finally, let $r_{i} \sim$ const. $i$. When $M_{1}=1$, the conditions (1.4) and (1.1) are the same. When $M_{1}<1$, we have

$$
\int_{0}^{1} \frac{1-h(s)}{p(s)-s} d s<\infty \Longleftrightarrow \int_{0}^{1} \frac{1-h(s)}{1-s} d s<\infty .
$$

Notice that

$$
\int_{0}^{1} \frac{1-h(s)}{1-s} d s=\sum_{j=1}^{\infty} h_{j} \sum_{\ell=0}^{j-1} \int_{0}^{1} s^{\ell} d s=\sum_{j=1}^{\infty} h_{j} \sum_{\ell=0}^{j-1} \frac{1}{\ell+1} .
$$

We have

$$
\int_{0}^{1} \frac{1-h(s)}{1-s} d s<\infty \Longleftrightarrow \sum_{j=1}^{\infty} h_{j} \log j<\infty .
$$

Thus, when $M_{1}<1$, the last assertion of Theorem 1.4 now follows from (1.4).

d) The last assertion of the theorem follows from [5; Corollary 4.49].

We have just proved that when $r_{i} \sim$ const. $i^{\theta}(i, \theta \geq 1)$, the process is positive recurrent iff (1.4) holds. On the other hand, part (1) of Theorem 1.4 says that the process is positive recurrent whenever $\theta>2$. To prove the consistence of these two conclusions, one has to show that (1.4) holds for all $\theta>2$. The case where $M_{1}<1$ is simple:

$$
\int_{0}^{1} \frac{1-h(s)}{p(s)-s}(1-s)^{\theta-1} d s \leq \int_{0}^{1} \frac{(1-s)^{\theta-1}}{p(s)-s} d s \leq \text { const. } \int_{0}^{1}(1-s)^{\theta-2} d s<\infty .
$$

When $M_{1}=1$, let $\tilde{p}(s)=p_{0}+\left(1-2 p_{0}\right) s+p_{0} s^{2}$. Then, it is easy to check that $p(s) \geq \tilde{p}(s)$, $0 \leq s<1$. Thus we have

$$
\begin{aligned}
\int_{0}^{1} \frac{1-h(s)}{p(s)-s}(1-s)^{\theta-1} d s & \leq \int_{0}^{1} \frac{1-h(s)}{\tilde{p}(s)-s}(1-s)^{\theta-1} d s \\
& =\frac{1}{p_{0}} \sum_{j=1}^{\infty} h_{j} \int_{0}^{1}\left(1-s^{j}\right)(1-s)^{\theta-3} d s \\
& =\frac{1}{p_{0}} \sum_{j=1}^{\infty} h_{j}\left(\frac{1}{\theta-2}-\frac{j}{\theta-2} B(j, \theta-1)\right) .
\end{aligned}
$$

By Stirling formula, one can check that the integral is finite.

Acknowledgement. The author would like to acknowledge Prof. Mu-Fa Chen and Prof. Guang-Lu Gong for their guidance and encouragement during the writing of the paper, and 


\section{REFERENCES}

[1] Anderson, W. J. (1991), Continuous-Time Markov Chains, Springer-Verlag.

[2] Asmussen, S. and Hering, H. (1983), Branching Processes, Birkhäuser.

[3] Athreya, K. B. and Ney, P. E. (1972), Branching Processes, Springer-Verlag.

[4] Chen, M. F. (1991), On three classical problem for Markov chains with continuous time parameters, J. Appl. Prob. 28, 305-320.

[5] Chen, M. F. (1992), From Markov Chains to Non-Equilibrium Particle Systems, World Scientific.

[6] Gao, S. Z. (1992), Linear controlled branching processes (In Chinese), Wuhan J. Math. 92:4, 415-422.

[7] Harris, T. E. (1963), Branching Processes, Springer-Verlag.

[8] Hou, Z. T. and Guo, Q. F.(1978), Time-Homogeneous Markov Processes with Countable State Space (In Chinese), Beijing Sci. Press, English translation (1988), Beijing Sci. Press and Springer-Verlag.

[9] Pakes, A. G. and Tavaré, S. (1981), Comments on the age distribution of Markov processes, 13, 681-703, Adv. Appl. Prob.

[10] Yamazato, M. (1975), Some results on continuous time branching process with state-dependent immigration, 17, 479-497, J. Math. Soc. Japan.

[11] Yan, S. J. and Chen, M. F. (1986), Multidimensional Q-processes, 7(B) : 1, 90-110, Chin. Ann. Math. 